\title{
Some Remarks on Convolution of Collection Integrals
}

\author{
Adam Šeliga \\ Faculty of Civil Engineering, Slovak University of Technology, \\ Radlinského 11, 81005 Bratislava, Slovakia, \\ adam.seliga@stuba.sk
}

\begin{abstract}
In this paper, we revisit the definition of a convolution of aggregation functions and we will examine the convolution of collection integrals defined on a finite space. Also, we introduce the concept of a convolution and of a lower convolution for monotone measures and examine properties of these convolutions with respect to collection integrals. Some concluding remarks are added.
\end{abstract}

Keywords: Convolution, Collection integral, Aggregation functions, Monotone measures

\section{Introduction}

Aggregation functions are a blooming part of mathematics which attracts many mathematicians and researchers both from the pure and the applied branches.

In this contribution, we revisit the definition of convolution of aggregation functions given in [7]. Convolution is a process of merging two objects of the same type into another such object. This idea found its place both in the theory, e.g., in functional analysis, and in the practice, e.g., in computer vision.

The theory of non-linear integrals has many applications, including the multi-criteria decision making, or the game theory. One class of non-linear integrals is the class of collection integrals introduced in [9] which is a special case of a more general framework, the framework of decomposition integrals $[3,8]$.

In this paper we examine the interaction of convolutions and collection integrals which can be viewed as aggregation functions and also we introduce and examine the convolution of monotone measures. The rest of the paper is organized as follows. In Section 2, some preliminary notions and definitions are given.
Section 3 is devoted to the examination of the convolution of collection integrals, including self-convolution. In Section 4, the convolution and the lower convolution of monotone measures is introduced and some of their properties are listed. In the last section, some concluding remarks and a sketch of the future research are given.

\section{Preliminaries}

Throughout the paper we will consider a finite nonempty space $X$ and, in this setting, we may assume that $X=\{1,2, \ldots, n\}$ for some $n \in \mathbb{N}$.

A function is any mapping $X \rightarrow[0, \infty[$ and the class of all functions will be denoted by $\mathbb{F}$. A monotone measure is a set function $\mu: 2^{X} \rightarrow[0, \infty$ [ that is grounded, i.e., $\mu(\varnothing)=0$, and monotone with respect to set inclusion, i.e., for any $A \subseteq B \subseteq X$ we have $\mu(A) \leq \mu(B)$. The class of all monotone measures will be denoted by $\mathbb{M}$. A monotone measure $\mu \in \mathbb{M}$ is called super-additive if and only if

$$
\mu(A \cup B) \geq \mu(A)+\mu(B)
$$

holds for all disjoint sets $A, B \subseteq X$. Analogously, the monotone measure $\mu$ is called sub-additive if and only if

$$
\mu(A \cup B) \leq \mu(A)+\mu(B)
$$

for all disjoint sets $A, B \subseteq X$.

A collection is any non-empty subset of $2^{X} \backslash\{\varnothing\}$. The class of all collections is denoted by $\mathbb{D}$.

Let $\left(\alpha_{A}\right)_{A \in \mathcal{D}}$ be a sequence of non-negative real numbers, where $\mathcal{D}$ is some collection. We say that $\left(\alpha_{A}\right)_{A \in \mathcal{D}}$ is a sub-decomposition of a function $f \in \mathbb{F}$ in $\mathcal{D}$ if and only if

$$
\sum_{A \in \mathcal{D}} \alpha_{A} \mathbf{1}_{A} \leq f,
$$

where $\mathbf{1}_{A} \in \mathbb{F}$ is the indicator function of the set $A \subseteq X$. 
A collection integral [9] with respect to a collection $\mathcal{D} \in \mathbb{D}$ and a monotone measure $\mu \in \mathbb{M}$ is an operator

$$
\operatorname{col}_{\mathcal{D}}^{\mu}: \mathbb{F} \rightarrow[0, \infty[
$$

such that its value for a function $f \in \mathbb{F}$ is given by

$$
\bigvee\left\{\sum_{A \in \mathcal{D}} \alpha_{A} \mu(A):\left(\alpha_{A}\right)_{A \in \mathcal{D}} \text { is a sub-decomp. of } f\right\} .
$$

Collection integrals are a special sub-class of decomposition integrals $[3,8]$ with respect to singleton decomposition systems.

Some examples of collection integrals are the chain integral [9], if $\mathcal{D}$ forms a chain (with respect to set inclusion), or the concave integral [5] of Lehrer.

Note that any function $f$ can be represented as an $n$ tuple of values $(f(1), f(2), \ldots, f(n))$. In this setting, collection integrals can be viewed as aggregation functions. An aggregation function $[1,4,6]$ on $[0, \infty[$ is a mapping

$$
\mathrm{A}:\left[0, \infty\left[^{n} \rightarrow[0, \infty[\right.\right.
$$

such that $A$ is grounded, i.e., $A(\mathbf{0})=0$, and $A$ is nondecreasing, i.e., for $\mathbf{x} \leq \mathbf{y}$ one has $A(\mathbf{x}) \leq A(\mathbf{y})$. The class of all aggregation functions will be denoted by the symbol $\mathbb{A}$.

In the paper [7], we have introduced a concept of convolution of aggregation functions, more specifically, four different types of convolutions. In this contribution we will be interested only in the (upper) convolution.

Let $A, B \in \mathbb{A}$ be two aggregation functions. Their convolution is an aggregation function $A \nabla B$ given by

$$
(A \nabla B)(\mathbf{x})=\bigvee_{\mathbf{0} \leq \mathbf{t} \leq \mathbf{x}}(A(\mathbf{t})+B(\mathbf{x}-\mathbf{t}))
$$

for all $\mathbf{x} \in\left[0, \infty\left[{ }^{n}\right.\right.$.

\section{Collection integrals and convolution}

In this part we will examine the convolution of collection integrals. We start with collection integrals with respect to the same monotone measure.

Proposition 1. Let $\mathcal{D}_{1}, \mathcal{D}_{2} \in \mathbb{D}$ be two collections. Then

$$
\operatorname{col}_{\mathcal{D}_{1}}^{\mu} \nabla \operatorname{col}_{\mathcal{D}_{2}}^{\mu}=\operatorname{col}_{\mathcal{D}_{1} \cup \mathcal{D}_{2}}^{\mu}
$$

for any monotone measure $\mu \in \mathbb{M}$.

Proof. Let $f \in \mathbb{F}$ be any function. Then

$$
\left(\operatorname{col}_{\mathcal{D}_{1}}^{\mu} \nabla \operatorname{col}_{\mathcal{D}_{2}}^{\mu}\right)(f)=\bigvee_{\substack{g \in \mathbb{F} \\ g \leq f}}\left(\operatorname{col}_{\mathcal{D}_{1}}^{\mu}(g)+\operatorname{col}_{\mathcal{D}_{2}}^{\mu}(f-g)\right),
$$

i.e., due to the finiteness of $X$, there exists $\bar{g} \in \mathbb{F}, \bar{g} \leq f$, such that

$$
\left(\operatorname{col}_{\mathcal{D}_{1}}^{\mu} \nabla \operatorname{col}_{\mathcal{D}_{2}}^{\mu}\right)(f)=\operatorname{col}_{\mathcal{D}_{1}}^{\mu}(\bar{g})+\operatorname{col}_{\mathcal{D}_{2}}^{\mu}(f-\bar{g}) .
$$

But then, there are sub-decompositions $\left\{\alpha_{A}\right\}_{A \in \mathcal{D}_{1}}$ of $\bar{g}$ in $\mathcal{D}_{1}$ and $\left\{\beta_{B}\right\}_{B \in \mathcal{D}_{2}}$ of $(f-\bar{g})$ in $\mathcal{D}_{2}$ such that

$$
\operatorname{col}_{\mathcal{D}_{1}}^{\mu}(\bar{g})=\sum_{A \in \mathcal{D}_{1}} \alpha_{A} \mu(A),
$$

and

$$
\operatorname{col}_{\mathcal{D}_{2}}^{\mu}(f-\bar{g})=\sum_{B \in \mathcal{D}_{2}} \beta_{B} \mu(B) .
$$

Summing these two sub-decompositions we obtain a sub-decomposition $\left(\gamma_{C}\right)_{C \in \mathcal{D}_{1} \cup \mathcal{D}_{2}}$

$$
\gamma_{C}= \begin{cases}\alpha_{C}, & \text { if } C \in \mathcal{D}_{1} \backslash \mathcal{D}_{2}, \\ \beta_{C}, & \text { if } C \in \mathcal{D}_{2} \backslash \mathcal{D}_{1}, \\ \alpha_{C}+\beta_{C}, & \text { if } C \in \mathcal{D}_{1} \cap \mathcal{D}_{2},\end{cases}
$$

of $f$ in $\mathcal{D}_{1} \cup \mathcal{D}_{2}$ which implies that

$$
\begin{aligned}
\sum_{C \in \mathcal{D}_{1} \cup \mathcal{D}_{2}} \gamma_{C} \mu(C) & =\sum_{A \in \mathcal{D}_{1}} \alpha_{A} \mu(A)+\sum_{B \in \mathcal{D}_{2}} \beta_{B} \mu(B) \\
& \leq \operatorname{col}_{\mathcal{D}_{1} \cup \mathcal{D}_{2}}^{\mu}(f)
\end{aligned}
$$

from which we obtain

$$
\operatorname{col}_{\mathcal{D}_{1}}^{\mu}(\bar{g})+\operatorname{col}_{\mathcal{D}_{2}}^{\mu}(f-\bar{g}) \leq \operatorname{col}_{\mathcal{D}_{1} \cup \mathcal{D}_{2}}^{\mu}(f),
$$

i.e.,

$$
\left(\operatorname{col}_{\mathcal{D}_{1}}^{\mu} \nabla \operatorname{col}_{\mathcal{D}_{2}}^{\mu}\right)(f) \leq \operatorname{col}_{\mathcal{D}_{1} \cup \mathcal{D}_{2}}^{\mu}(f) .
$$

Now we need to prove the reversed inequality. Let

$$
\sum_{A \in \mathcal{D}_{1} \cup \mathcal{D}_{2}} \gamma_{A} \mathbf{1}_{A}
$$

be a sub-decomposition of $f$ such that

$$
\operatorname{col}_{\mathcal{D}_{1} \cup \mathcal{D}_{2}}^{\mu}(f)=\sum_{A \in \mathcal{D}_{1} \cup \mathcal{D}_{2}} \gamma_{A} \mu(A) .
$$

Then we can split the sum into two sums while obtaining

$$
\operatorname{col}_{\mathcal{D}_{1} \cup \mathcal{D}_{2}}^{\mu}(f)=\sum_{A \in \mathcal{D}_{1}} \gamma_{A} \mu(A)+\sum_{A \in \mathcal{D}_{2} \backslash \mathcal{D}_{1}} \gamma_{A} \mu(A) .
$$

Now, let us set

$$
g=\sum_{A \in \mathcal{D}_{1}} \gamma_{A} \mathbf{1}_{A}
$$

Then, the first sum in (2) is a sub-decomposition of $g$ in $\mathcal{D}_{1}$ and the second sum is a sub-decomposition of $(f-g)$ in $\mathcal{D}_{2}$. In other words, we obtain that

$$
\operatorname{col}_{\mathcal{D}_{1} \cup \mathcal{D}_{2}}^{\mu}(f) \leq \operatorname{col}_{\mathcal{D}_{1}}^{\mu}(g)+\operatorname{col}_{\mathcal{D}_{2}}^{\mu}(f-g)
$$


for some function $g \in \mathbb{F}$ such that $g \leq f$. But this implies that

$$
\operatorname{col}_{\mathcal{D}_{1} \cup \mathcal{D}_{2}}^{\mu}(f) \leq\left(\operatorname{col}_{\mathcal{D}_{1}}^{\mu} \nabla \operatorname{col}_{\mathcal{D}_{2}}^{\mu}\right)(f)
$$

which implies that, in a combination with (1), that

$$
\operatorname{col}_{\mathcal{D}_{1} \cup \mathcal{D}_{2}}^{\mu}(f)=\left(\operatorname{col}_{\mathcal{D}_{1}}^{\mu} \nabla \operatorname{col}{ }_{\mathcal{D}_{2}}^{\mu}\right)(f) \text {. }
$$

Because the choice of the function $f \in \mathbb{F}$ was arbitrary, the equality holds in general.

Now we will turn our attention to the convolution of collection integrals with respect to the same collection.

Proposition 2. Let $\mu_{1}, \mu_{2} \in \mathbb{M}$ be two monotone measures. Then

$$
\operatorname{col}_{\mathcal{D}}^{\mu_{1}} \nabla \operatorname{col}_{\mathcal{D}}^{\mu_{2}}=\operatorname{col}_{\mathcal{D}}^{\mu_{1} \vee \mu_{2}}
$$

for any collection $\mathcal{D} \in \mathbb{D}$.

Proof. The proof is similar to the proof of Proposition 1 and thus omitted.

Remark 1. It is not so hard to notice, e.g., from the monotonicity of the collection integrals and the convolution, that

$$
\operatorname{col}_{\mathcal{D}_{1}}^{\mu_{1}} \nabla \operatorname{col}_{\mathcal{D}_{2}}^{\mu_{2}} \leq \operatorname{col}_{\mathcal{D}_{1} \cup \mathcal{D}_{2}}^{\mu_{1} \vee \mu_{2}},
$$

in general. The previous two propositions could indicate that

$$
\operatorname{col}_{\mathcal{D}_{1}}^{\mu_{1}} \nabla \operatorname{col}_{\mathcal{D}_{2}}^{\mu_{2}}=\operatorname{col}_{\mathcal{D}_{1} \cup \mathcal{D}_{2}}^{\mu_{1} \vee \mu_{2}}
$$

also holds in general. This is not the case, see the following example.

Example 1. Consider the space $X=\{1,2\}$, collections $\mathcal{D}_{1}=\{\{1,2\},\{1\}\}$ and $\mathcal{D}_{2}=\{\{2\}\}$, and monotone measures $\mu_{1}, \mu_{2} \in \mathbb{M}$ with values given in Table 1 . Then we obtain that

$$
\operatorname{col}_{\mathcal{D}_{1}}^{\mu_{1}}\left(g_{1}, g_{2}\right)=g_{1}
$$

and

$$
\operatorname{col}_{\mathcal{D}_{2}}^{\mu_{2}}\left(f_{1}-g_{1}, f_{2}-g_{2}\right)=f_{2}-g_{2} .
$$

Then, e.g.

$$
\left(\operatorname{col}_{\mathcal{D}_{1}}^{\mu_{1}} \nabla \operatorname{col}_{\mathcal{D}_{2}}^{\mu_{2}}\right)(1,2)=\bigvee_{\substack{g_{1} \in[0,1] \\ g_{2} \in[0,2]}}\left(g_{1}+2-g_{2}\right)=3 .
$$

But,

$$
\operatorname{col}_{\mathcal{D}_{1} \cup \mathcal{D}_{2}}^{\mu_{1} \vee \mu_{2}}(1,2)=11
$$

which shows that

$$
\left(\operatorname{col}_{\mathcal{D}_{1}}^{\mu_{1}} \nabla \operatorname{col}_{\mathcal{D}_{2}}^{\mu_{2}}\right)(f) \neq \operatorname{col}_{\mathcal{D}_{1} \cup \mathcal{D}_{2}}^{\mu_{1} \vee \mu_{2}}(f),
$$

in general.

\begin{tabular}{|c|c|c|c|c|}
\hline & $\varnothing$ & $\{1\}$ & $\{2\}$ & $\{1,2\}$ \\
\hline$\mu_{1}$ & 0 & 1 & 1 & 1 \\
$\mu_{2}$ & 0 & 1 & 1 & 10 \\
\hline$\mu_{1} \vee \mu_{2}$ & 0 & 1 & 1 & 10 \\
\hline
\end{tabular}

Table 1: The values of monotone measures $\mu_{1}, \mu_{2}$, and $\mu_{1} \vee \mu_{2}=\mu_{2}$ used in Example 1.

In the following proposition, a sufficient condition to ensure the equality

$$
\operatorname{col}_{\mathcal{D}_{1}}^{\mu_{1}} \nabla \operatorname{col}_{\mathcal{D}_{2}}^{\mu_{2}}=\operatorname{col}_{\mathcal{D}_{1} \cup \mathcal{D}_{2}}^{\mu_{1} \vee \mu_{2}}
$$

is given.

Proposition 3. Let $\mu_{1}, \mu_{2} \in \mathbb{M}$ be two monotone measures and let $\mathcal{D}_{1}, \mathcal{D}_{2} \in \mathbb{D}$ be two collections. If

$$
\mu_{1}(A) \geq \mu_{2}(A) \text { for all } A \in \mathcal{D}_{1}
$$

and

$$
\mu_{1}(A) \leq \mu_{2}(A) \text { for all } A \in \mathcal{D}_{2}
$$

then

$$
\operatorname{col}_{\mathcal{D}_{1}}^{\mu_{1}} \nabla \operatorname{col}_{\mathcal{D}_{2}}^{\mu_{2}}=\operatorname{col}_{\mathcal{D}_{1} \cup \mathcal{D}_{2}}^{\mu_{1} \vee \mu_{2}}
$$

Proof. From the stated conditions, we obtain that $\mu_{1}(A) \geq \mu_{2}(A)$ for all $A \in \mathcal{D}_{1} \backslash \mathcal{D}_{2}$, next $\mu_{2}(A) \geq \mu_{1}(A)$ for $A \in \mathcal{D}_{2} \backslash \mathcal{D}_{1}$, and $\mu_{1}(A)=\mu_{2}(A)$ for $A \in \mathcal{D}_{1} \cap \mathcal{D}_{2}$. Because of that one obtains that

$$
\operatorname{col}_{\mathcal{D}_{1}}^{\mu_{1}}=\operatorname{col}_{\mathcal{D}_{1}}^{\mu_{1} \vee \mu_{2}} \text { and } \operatorname{col}_{\mathcal{D}_{2}}^{\mu_{2}}=\operatorname{col}_{\mathcal{D}_{2}}^{\mu_{1} \vee \mu_{2}}
$$

and following Proposition 1 we obtain the desired result.

Remark 2. From the previous propositions it clearly follows that

$$
\operatorname{col}_{\mathcal{D}}^{\mu} \nabla \operatorname{col}_{\mathcal{D}}^{\mu}=\operatorname{col}_{\mathcal{D}}^{\mu}
$$

for any collection $\mathcal{D} \in \mathbb{D}$ and any monotone measure $\mu \in \mathbb{M}$. This is also a result of the super-additivity of collection integrals and properties of the convolution. We refer the interested reader to papers $[2,7]$.

\section{Convolution of monotone measures}

Now we can turn our attention to the convolution of monotone measures. We propose the following definition of convolution.

Definition 1. Let $\mu, v \in \mathbb{M}$ be two monotone measures. Their convolution is a set function $\mu \nabla v: 2^{X} \rightarrow[0, \infty[$ given by

$$
(\mu \nabla v)(A)=\sup _{B \subseteq A}(\mu(B)+v(A \backslash B))
$$

for all $A \in 2^{X}$. 
Proposition 4. The convolution of two monotone measures $\mu, v \in \mathbb{M}$ is again a monotone measure.

Proof. It is not hard to see that $(\mu \nabla v)(\varnothing)=0$. It remains to show the monotonicity of the convolution. Let $A, A^{\prime}$ be two sets such that $A \subseteq A^{\prime}$. Then for any $B \subseteq A$ we have that

$$
\mu(B)+v(A \backslash B) \leq \mu(B)+v\left(A^{\prime} \backslash B\right)
$$

which implies that

$$
\begin{aligned}
(\mu \nabla v)(A) & \leq \sup _{B \subseteq A}\left(\mu(B)+v\left(A^{\prime} \backslash B\right)\right) \\
& \leq \sup _{B \subseteq A^{\prime}}\left(\mu(B)+v\left(A^{\prime} \backslash B\right)\right)=(\mu \nabla v)\left(A^{\prime}\right) .
\end{aligned}
$$

Because $A$ and $A^{\prime}$ were arbitrary, the proposition follows.

Directly from the definition of the convolution we have the following result.

Corollary 1. $\mu \nabla v \geq \mu \vee v$.

The following is the corollary concerning collection integrals with respect to the convolution of two monotone measures.

Corollary 2. Let $\mu, v \in \mathbb{M}$ be two monotone measures and let $\mathcal{D} \in \mathbb{D}$ be a collection. Then

$$
\operatorname{col}_{\mathcal{D}}^{\mu \nabla v} \geq \operatorname{col}_{\mathcal{D}}^{\mu} \vee \operatorname{col}_{\mathcal{D}}^{v}
$$

We can obtain also the following result concerning the super-additivity of monotone measures.

Proposition 5. A monotone measure $\mu \in \mathbb{M}$ is superadditive if and only if $\mu \nabla \mu=\mu$.

Proof. From the super-additivity we have that

$$
\mu(B)+\mu(A \backslash B) \leq \mu(A)
$$

for any sets $A, B$ such that $B \subseteq A$. This trivially implies that $(\mu \nabla \mu)(A)=\mu(A)$ holds for any $A \subseteq X$. Now the proof of the contrary. Let us assume that $\mu \nabla \mu=\mu$ and let $A, B \subseteq X$ be two disjoint sets. We need to prove that $\mu(A \cup B) \geq \mu(A)+\mu(B)$. This follows from the fact that

$$
\begin{aligned}
\mu(A \cup B) & =(\mu \nabla \mu)(A \cup B) \\
& =\sup _{Z \subseteq A \cup B}(\mu(Z)+\mu((A \cup B) \backslash Z)) \\
& \geq \mu(B)+\mu(A)
\end{aligned}
$$

choosing $Z=B$. The monotone measure $\mu$ is thus super-additive and the proposition follows.
As in the case of the convolution of aggregation functions, also a lower convolution of monotone measures can be introduced.

Definition 2. Let $\mu, v \in \mathbb{M}$ be two monotone measures. $A$ lower convolution of monotone measures $\mu$ and $v$ is a set function $\mu \triangle v: 2^{X} \rightarrow[0, \infty[$ defined by

$$
(\mu \triangle v)(A)=\inf _{B \subseteq A}(\mu(B)+v(A \backslash B))
$$

for all $A \in 2^{X}$.

As in the case of the (upper) convolution of monotone measures, we obtain the following properties for the lower convolution.

Proposition 6. Let $\mu, v \in \mathbb{M}$ be two monotone measures. Then (i) $\mu \triangle v$ is also a monotone measure; (ii) $\mu \Delta v \leq \mu \wedge v$; and (iii) $\mu \triangle \mu=\mu$ if and only if $\mu$ is sub-additive monotone measure.

Proof. (i) It is easy to notice that $(\mu \triangle v)(\varnothing)=0$ holds. For the monotonicity, let $A, A^{\prime} \in 2^{X}$ be any sets such that $A \subseteq A^{\prime}$. The monotonicity of $\mu \triangle v$ is proved by the following:

$$
\begin{aligned}
(\mu \triangle v)\left(A^{\prime}\right) & =\inf _{B \subseteq A^{\prime}}\left(\mu(B)+v\left(A^{\prime} \backslash B\right)\right) \\
& \geq \inf _{B \subseteq A^{\prime}}\left(\mu(B \cap A)+v\left(\left(A^{\prime} \backslash B\right) \cap A\right)\right) \\
& =\inf _{B \subseteq A}(\mu(B)+v(A \backslash B))=(\mu \triangle v)(A) .
\end{aligned}
$$

Because $A$ and $A^{\prime}$ were arbitrary, the monotonicity follows. Proofs of (ii) and (iii) are analogous to proofs of Corollary 1 and Proposition 5.

For the collection integrals we obtain the following result.

Corollary 3. Let $\mu, v \in \mathbb{M}$ be two monotone measures and let $\mathcal{D} \in \mathbb{D}$ be a collection. Then

$$
\operatorname{col}_{\mathcal{D}}^{\mu \Delta v} \leq \operatorname{col}_{\mathcal{D}}^{\mu} \wedge \operatorname{col}_{\mathcal{D}}^{v}
$$

Remark 3. Based on the Corollaries 2 and 3 we have the following chain of inequalities:

$$
\operatorname{col}_{\mathcal{D}}^{\mu \Delta v} \leq \operatorname{col}_{\mathcal{D}}^{\mu} \wedge \operatorname{col}_{\mathcal{D}}^{v} \leq \operatorname{col}_{\mathcal{D}}^{\mu} \vee \operatorname{col}_{\mathcal{D}}^{v} \leq \operatorname{col}_{\mathcal{D}}^{\mu \nabla v}
$$

for all monotone measures $\mu, v \in \mathbb{M}$ and all collections $\mathcal{D} \in \mathbb{D}$.

Remark 4. We can observe some algebraic properties of operations $\nabla$ and $\triangle$. In particular, both $(\mathbb{M}, \nabla)$ and $(\mathbb{M}, \triangle)$ are abelian semigroups. Concerning $(\mathbb{M}, \nabla)$, nullary measure $\mu_{o} \in \mathbb{M}, \mu_{o}(A)=0$ for every $A \subseteq X$, is a neutral element, and $\mu \in \mathbb{M}$ is an idempotent element if and only if $\mu$ is super-additive. Similarly, for $(\mathbb{M}, \triangle)$, $\mu_{o}$ is the zero element and its idempotent elements are just the sub-additive measures. 


\section{Concluding remarks}

In this work we examined some properties of the convolution of collection integrals and the convolution of monotone measures. In the future research we plan to disseminate results in this area, turning our attention also to decomposition integrals and also applying super- and sub-convolutions both for decomposition integrals and also for monotone measures.

\section{Acknowledgement}

The author was supported by the Slovak Research and Development Agency under the contracts no. APVV17-0066 and no. APVV-18-0052. Also the support of the grant VEGA 1/0006/19 is kindly announced.

\section{References}

[1] G. Beliakov, A. Pradera, T. Calvo, Aggregation functions: a guide for practitioners, Vol. 221 of Studies in Fuzziness and Soft Computing, Springer, Berlin, 2007.

[2] Doria, S., Mesiar, R., Šeliga, A., A construction method of coherent lower and upper previsions based on collection integrals. Bollettino dell'Unione Matematica Italiana 13 (2020) 469476.

[3] Y. Even, E. Lehrer, Decomposition-integral: unifying Choquet and the concave integrals, Economic Theory 56 (2014) 33-58.

[4] M. Grabisch, J.L. Marichal, R. Mesiar, E. Pap, Aggregation functions, Encyclopedia of Mathematics, Cambridge University Press, Cambridge, 2009.

[5] E. Lehrer, A new integral for capacities, Economic Theory 39 (2009) 157-176.

[6] R. Mesiar, A. Kolesárová, A. Stupňanová, Quo vadis aggregation?, International Journal of General Systems 47(2) (2018) 97-117.

[7] R. Mesiar, A. Šeliga, A. Šipošová, and J. Širáň, Convolution of aggregation functions, International Journal of General Systems 49(7) (2020) 747-759.

[8] R. Mesiar, A. Stupňanová, Decomposition integrals, International Journal of Approximate Reasoning 54(8) (2013) 1252-1259.

[9] A. Šeliga, Decomposition integral without alternatives, its equivalence to Lebesgue integral, and computational algorithms. Journal of Automation, Mobile Robotics and Intelligent Systems 13(3) (2019) 41-48. 